\title{
Téoros
}

Revue de recherche en tourisme

\section{La réhabilitation urbaine, un produit touristique ?}

\section{Patrick Dieudonné}

Volume 21, numéro 2, été 2002

Patrimoines du $\mathrm{XX}^{\mathrm{e}}$ siècle

URI : https://id.erudit.org/iderudit/1072402ar

DOI : https://doi.org/10.7202/1072402ar

Aller au sommaire du numéro

Éditeur(s)

Université du Québec à Montréal

ISSN

0712-8657 (imprimé)

1923-2705 (numérique)

Découvrir la revue

Citer cet article

Dieudonné, P. (2002). La réhabilitation urbaine, un produit touristique?

Téoros, 21(2), 10-14. https://doi.org/10.7202/1072402ar d'utilisation que vous pouvez consulter en ligne.

https://apropos.erudit.org/fr/usagers/politique-dutilisation/ 


\section{La réhabilitation urbaine, un produit touristique?}

\section{Pałrick Dieudonné}

orsqu'en 1983 fut convoqué un colloque universitaire consacré aux reconstructions, l'équipe chargée de son organisation fut la première surprise par l'ampleur des réponses et leur variété internationale. Un simple regard sur l'histoire des villes, et même sur l'actualité, aurait certes montré que les destructions urbaines massives sont, hélas, la chose la mieux partagée au monde. Pour autant, pareille résonance offrait matière à questionnement. Dans les coulisses de ce premier colloque international des villes reconstruites, auquel allait succéder dix ans plus tard un rassemblement encore plus important, naquit une sorte de club informel, d'abord constitué par les élus et techniciens des villes de la façade atlantique, détruites au cours de la seconde guerre mondiale'.

Il n'était alors pas question de tourisme, même en Bretagne où cette activité est pourtant bien implantée. La seule évocation du nom de ces villes n'appelait encore que la commisération, quand ce n'était pas le sarcasme : grises, froides, sans âme, selon les termes d'une enquête menée auprès des Brestois sur l'image de leur ville, tels étaient les qualificatifs les plus communs pour en évoquer l'ambiance. Les offices de tourisme, pour qui le concept même de tourisme urbain n'était encore qu'embryonnaire, renvoyaient à d'autres produits plus séduisants de la campagne et du littoral les visiteurs qui avaient eu l'audace de venir se loger dans ces villes de grisaille. Seule Saint-Malo, portée par une tradition touristique ancienne, et par une reconstruction qui avait su en restituer le ca-

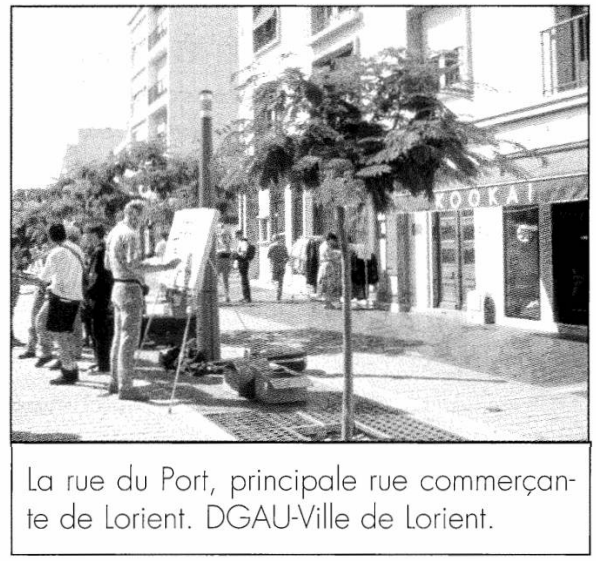

ractère pittoresque, semblait tirer son épingle du jeu, avec l'aide d'une puissante dénégation collective dont les vecteurs mériteraient une analyse spécifique : il n'est pas une publication consacrée à la « Bretagne historique » qui ne fasse une large place à cette silhouette urbaine dont la plupart des murs sont postérieurs à 1945 ...

\section{Rétablir la valeur des centres-villes}

Le club des villes reconstruites, qui allait se transformer en association formelle, se préoccupait d'un tout autre chantier que la valorisation touristique. Élus et techniciens se trouvaient confrontés à une dévalorisation accélérée du patrimoine immobilier des quartiers centraux, déserté par la population la plus aisée, et surtout dramatiquement négligé par la plupart des propriétaires. Les dispositifs de subventions n'avaient, de surcroît, pas prévu ce cas particulier, en réservant leurs budgets et leurs procédures techniques aux centres plus anciens. Il fallait donc, d'abord, inventer des protocoles de réhabilitation, dans les domaines administratif, tech- nique et financier, qui fussent à la hauteur de la tâche : dans la plupart de ces villes, plus de $80 \%$ du bâti appartenait à cette génération. Il fallait, de surcroît, convaincre de l'intérêt d'un investissement, car l'obsolescence des édifices n'était pas seulement visible; elle se doublait d'une inadéquation grandissante au mode de vie contemporain de l'organisation intérieure des immeubles, construits dans les années cinquante mais reproduisant le plus souvent le fonctionnement des logements du début du siècle.

Les plus grandes agglomérations, de Dunkerque, de Brest ou de Saint-Nazaire semblaient les mieux armées pour assurer un travail de cette ampleur. C'est pourtant de Lorient qu'est venu Le premier frémissement opérationnel est pourtant venu de Lorient, qui pouvait s'appuyer sur deux atouts. D'une part, le patrimoine architectural de la ville comptait quelques îlots rescapés, et notamment une architecture des années 1920-1930 dont la réhabilitation pouvait commencer à partir des dispositifs techniques existants. D'autre part, en raison de la taille de la ville (60 000 habitants

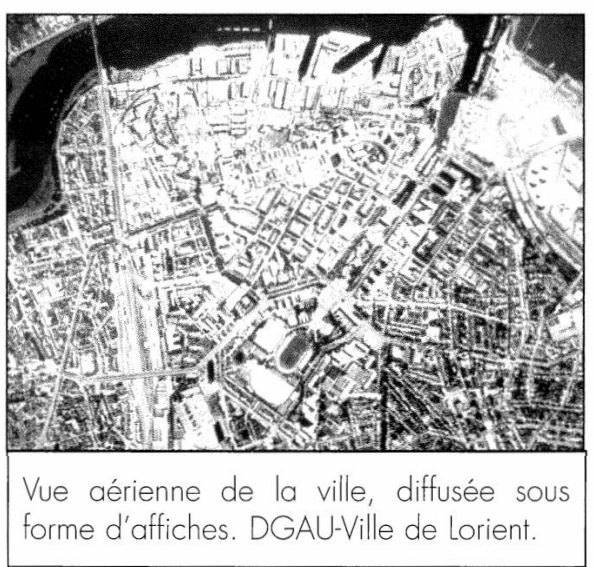




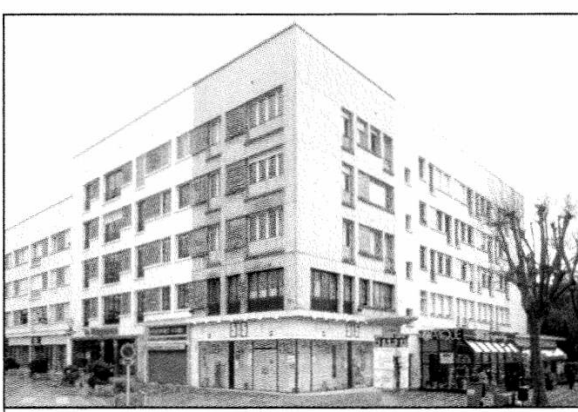

L'un des derniers immeubles non restaurés, témoin de l'état antérieur du bâti Photo : P. Dieudonné

aujourd'hui), un même service municipal assurait les fonctions de prospective et de réalisation des opérations, ce qui garantissait la souplesse indispensable dans un processus qui s'annonçait aussi long qu'expérimental.

Ce service d'urbanisme, la Direction générale de l'Aménagement urbain, fut très tôt conscient de la nécessité de mener, avant même les premiers chantiers, un travail de mémoire qui allait, quelques années plus tard, contaminer l'offre touristique. Mené avec les archives municipales et avec l'équipe universitaire qui préparait le second colloque international des villes reconstruites, ce travail donna d'emblée une tonalité particulière à la campagne de ravalement obligatoire ${ }^{2}$. Outre une étude conduite par un coloriste, plusieurs principes en guidèrent la mise en œuvre. L'opération devait renforcer de l'image du centre, permettre une nouvelle lecture de

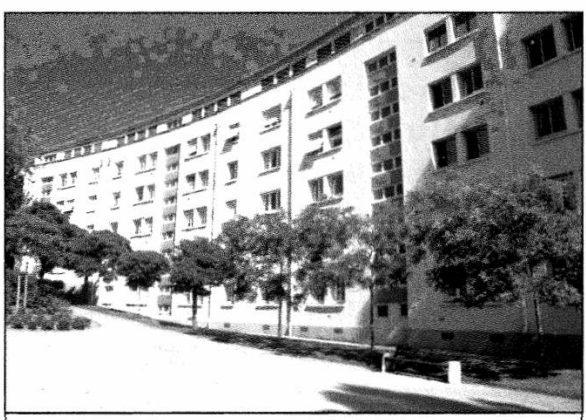

La "Banane ", immeuble courbe construit en 1953, est l'un des plus caractéristique de l'architecture lorientaise : volume simple - une barre courbe de 120 mètres porte-à-faux et toitures-terrasses, contrastes de l'enduit blanc et des détails de couleurs vives. Jean-Baptiste Hourlier, architecte. Photo : P. Dieudonné

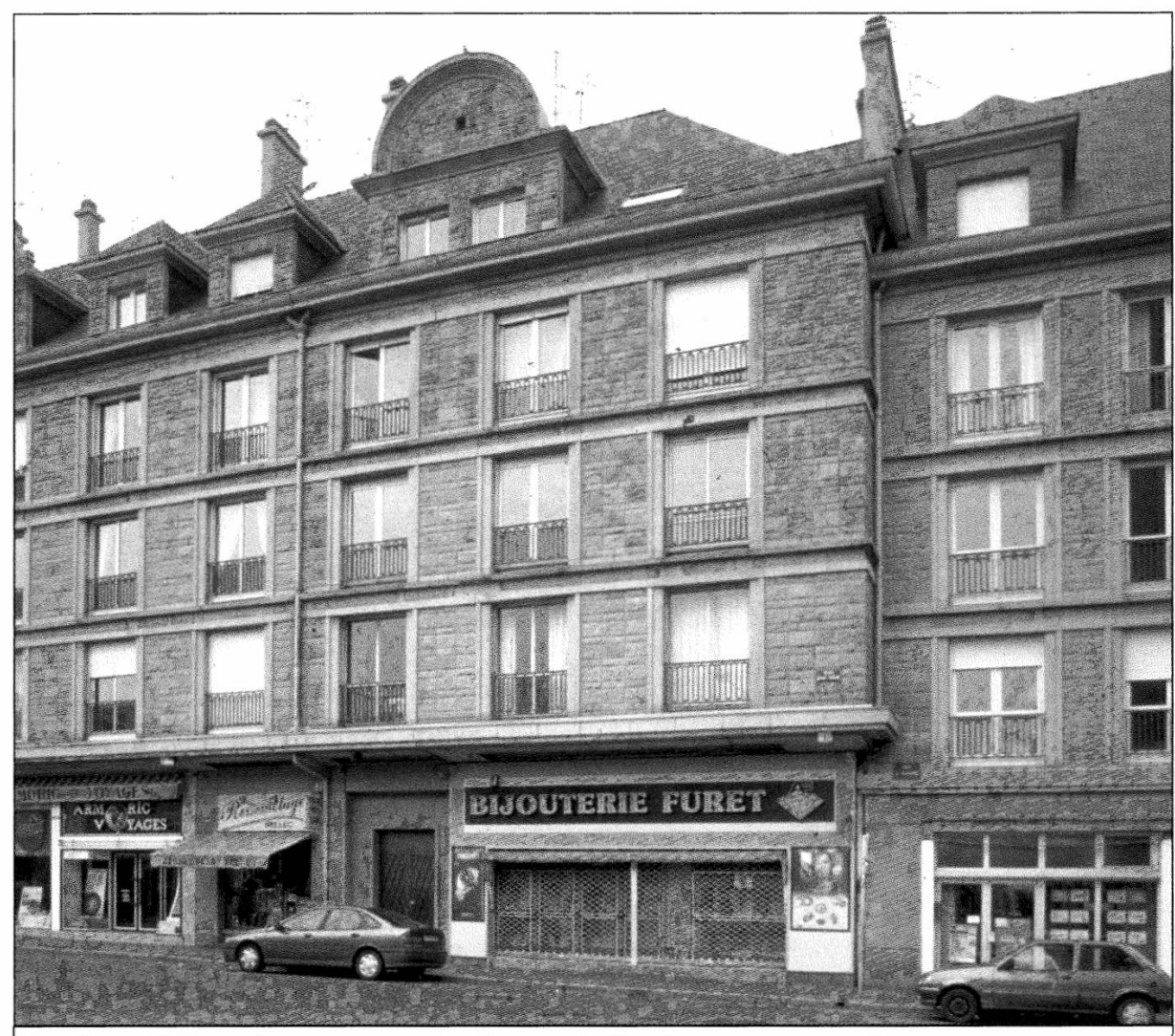

La place d'Alsace-Lorraine, une des formes de l'architecture régionaliste. Photo: P. Dieudonné.

l'architecture des années cinquante, être facile à réaliser pour l'ensemble des acteurs (techniciens municipaux, propriétaires, entrepreneurs du bâtiment), et surtout aboutir à une certaine pérennité des pratiques, car la municipalité ne souhaitait pas voir se reproduire, tous les trente ans, la même situation d'obsolescence. Les caractéristiques architecturales de la reconstruction orientèrent donc les réflexion sur le ravalement.

\section{Une architecture complexe et variée}

Cette architecture ressortit en effet à plusieurs catégories. Sa complexité est apparue au premier abord comme une difficulté, mais s'est révélée un atout important dans la mise en valeur d'une variété qui était masquée par un voile uniforme où se mêlaient la nostalgie de la ville détruite, l'image insipide de la reconstruction et l'état matériel, incontestablement détérioré, des immeubles. Outre la présence de quelques édifices d'avant-guerre, une partie du centre reconstruit repose sur une architecture sobre de maçonnerie enduite, rehaussée de quelques accents de modénature comme les larges bandeaux des «blocs-fenêtres ${ }^{3}$ » qui sont les signes stylistiques les plus saillants des années cinquante. Une discrète ferronnerie, aux motifs géométriques, vient parfois enrichir un travail architectural qui repose néanmoins, pour l'essentiel, sur l'expression de volumes simples. La variété, dans cette partie de la ville, provient en

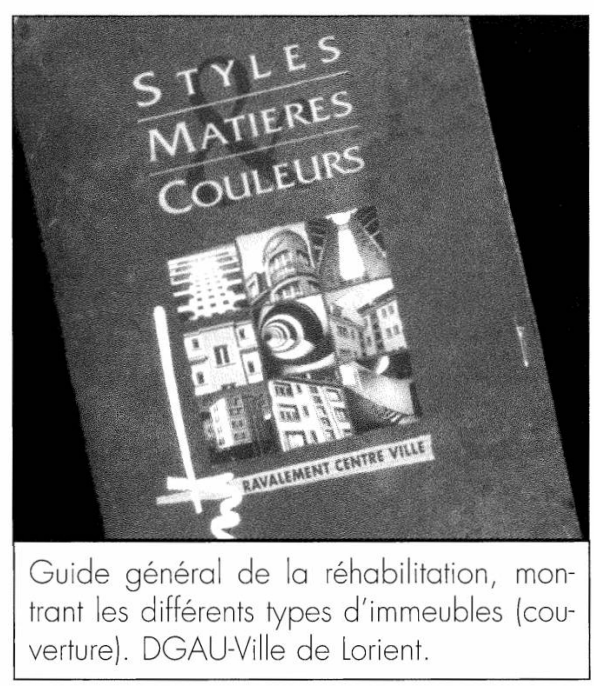




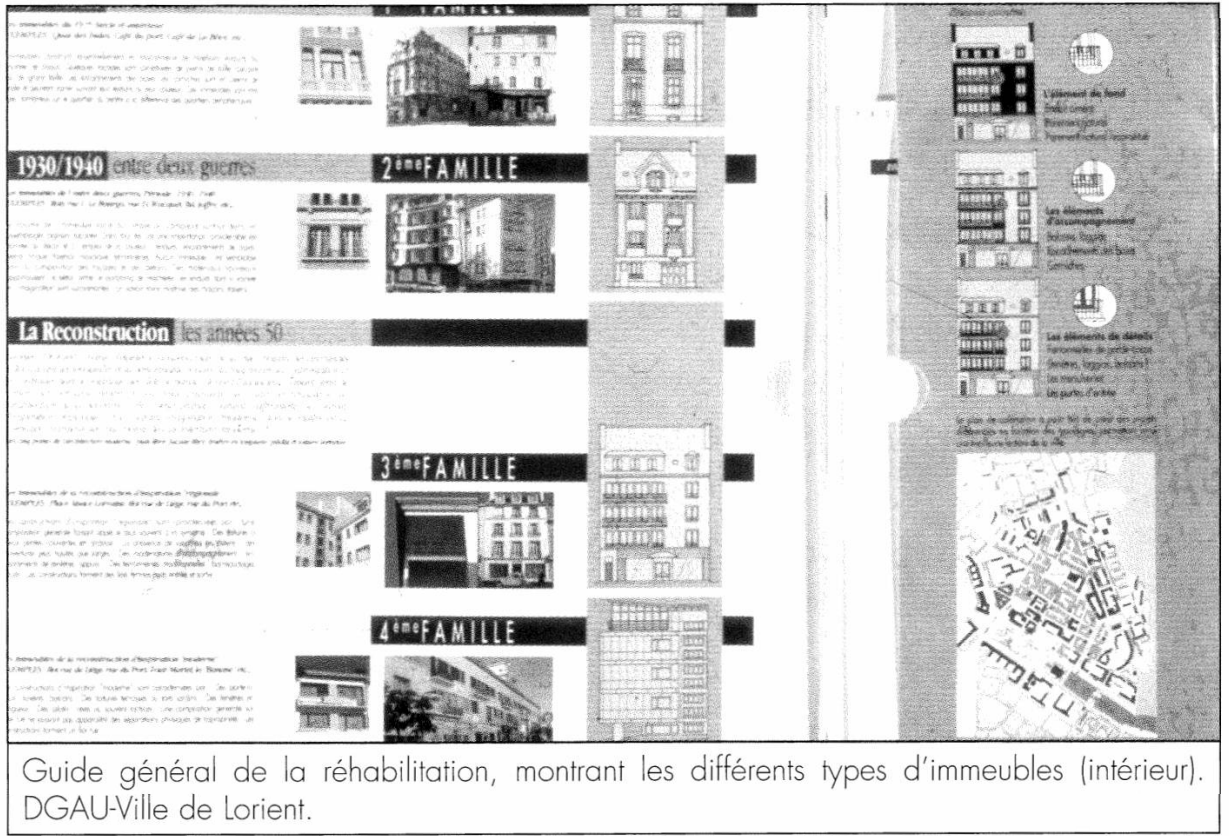

grande partie de l'inégalité des rythmes et des gabarits, que l'architecte en chef de la reconstruction avait autorisée, et parfois peut-être encouragée. En dépit de son autorité sur le plan général de la ville, Georges Tourry avait dû en effet composer avec les désirs des propriétaires et leurs moyens financiers, et il avait admis que ses confrères chargés de l'exécution des bâtiments puissent jouer pleinement le rôle dans l'expression architecturale.

Une seconde catégorie est représentée par une architecture dite « régionaliste ». Les attributs pittoresques dont elle se pare fréquemment ont été amendés pour cette transposition dans l'univers urbain, mais elle a conservé une partie de son vocabulaire, fait de matériaux locaux, de toits ardoisés à forte pente et de lucarnes rampantes. Les touristes les plus curieux peuvent même observer avec amusement des immeubles hybrides, régionalistes en façade et parfaitement modernes côté cour. Enfin, quelques édifices dégagent une silhouette exceptionnelle, celle de tours ou d'immeubles en barres de quelques étages, et constituent une troisième catégorie.

\section{Pédagogie et travail de mémoire}

En s'appuyant sur cette répartition, le service d'urbanisme construisit une doctrine de coloration reposant sur les caractéristiques typologiques des immeubles. Des guides explicatifs assortis de nuanciers spécifiques de chaque type furent mis à disposition des acteurs. Cette offre qui n'avait rien de touristique dépassa pourtant très vite son cadre d'origine, en raison notamment de l'extrême soin graphique dont ces guides avaient fait l'objet. Des documents de cette nature, qui devaient à la fois encadrer les choix et surtout convaincre les propriétaires, les entreprises du bâtiment et les contribuables du bien-fondé de cette action à grande échelle, ne pouvaient avoir d'efficacité que s'ils montraient de grandes qualités pédagogiques et un potentiel de séduction tout aussi probant. Il fallait apprendre à lire l'architecture dite « moderne », spontanément dénigrée par l'opinion publique, qui souffre ordinairement d'une image de marque détestable notamment parce que son vocabulaire est peu diffusé, si ce n'est sous l'angle de la caricature. L'indispensable travail d'explication est donc devenu, par la qualité des documents proposé, un atout de taille pour le tourisme et a donné à Lorient une avance considérable sur les autres villes reconstruites, qui sont à leur tour tentées par l'expérience mais utilisent des procédures notoirement différentes. La lourde approche de la ZPPAUP ${ }^{4}$, au Havre et à Brest, par exemple, obère le potentiel des campagnes de communication en raison des contraintes imposées par ce

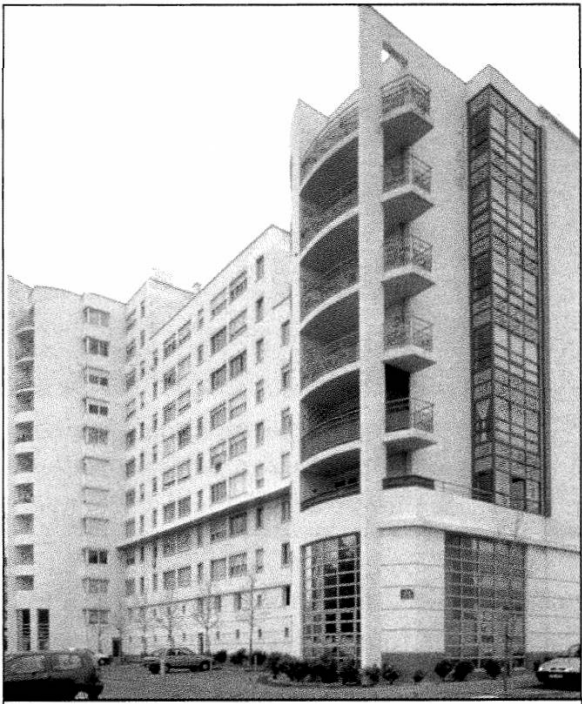

Quai de Rohan, opération de réhabilitation d'un grand ensemble de logements. L'immeuble a été coupé en deux parties pour laisser place à une rue, et sa façade a reçu de nouveaux éléments de confort (balcons, bow-windows, etc.)

Photo : P. Dieudonné

dispositif administratif, complexe et donc cantonné dans l'univers des spécialistes de l'architecture et du droit des sols.

Lorient décida de s'en tenir au cadre juridique existant, et se concentra sur les relations avec les différents acteurs de la ville. Des documents empruntant leur forme aux usages touristiques, cartes postales et affiches, furent même édités par le service d'urbanisme pour renforcer la campagne de réhabilitation, morale cette fois, de l'architecture lorientaise. Les concepteurs eurent l'intelligence de ne pas limiter leur collection à la seule architecture des années cinquante, mais renouèrent le fil de l'histoire, en proposant une série dont le parcours chronologique s'étendait sur tout le siècle, et jusqu'à l'actualité.

La démarche mise au point pour le ravalement du centre-ville allait en effet se propager à d'autres opérations : la réhabilitation d'un grand ensemble de logements - le quai de Rohan, transformé par l'architecte Roland Castro - , les aménagements d'espaces publics, et plus récemment reconversion de la base de sous-marins héritée de l'occupation allemande prirent place dans le même dispositif pédago- 


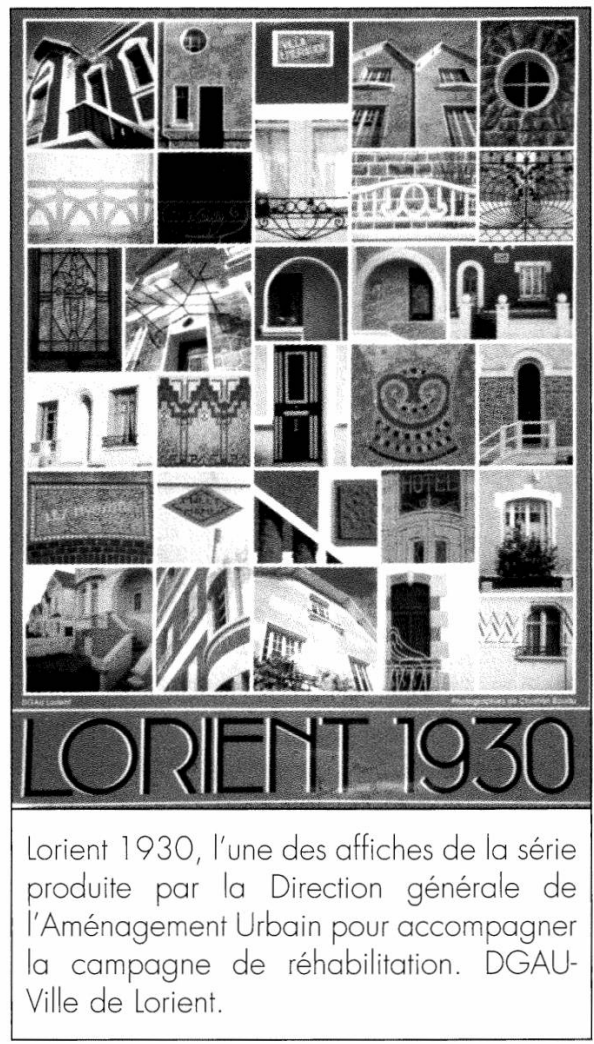

gique, et se trouvèrent rapidement inclus dans l'offre touristique lorientaise.

\section{L'intégration \\ à I'offre touristique}

De son côté, l'office de tourisme du pays de Lorient ${ }^{5}$, dont les préoccupations étaient en 1990 fort éloignées du tourisme urbain alors naissant, ne fut dans un premier temps qu'un simple relais des documents produits par le service d'urbanisme. Les cartes postales et affiches, notamment, rencontrèrent un grand succès auprès des Lorientais, au point qu'une certaine curiosité se manifesta dans l'office même pour une activité jusqu'alors négligée. L'office reprit donc la maîtrise de l'organisation des visites de la ville, qu'il avait depuis plusieurs années concédées à des agences privées. Parallèlement, la campagne de coloration et de réhabilitation des espaces publics portant ses fruits, des promotions spécifiques devenaient non seulement possibles, mais étaient même demandées par les habitants. Les éditeurs de cartes postales furent sollicités pour renouveler un fonds qui n'avait guère connu d'évolution depuis les années soixante, et une nouvelle image du centre commença de se propager.

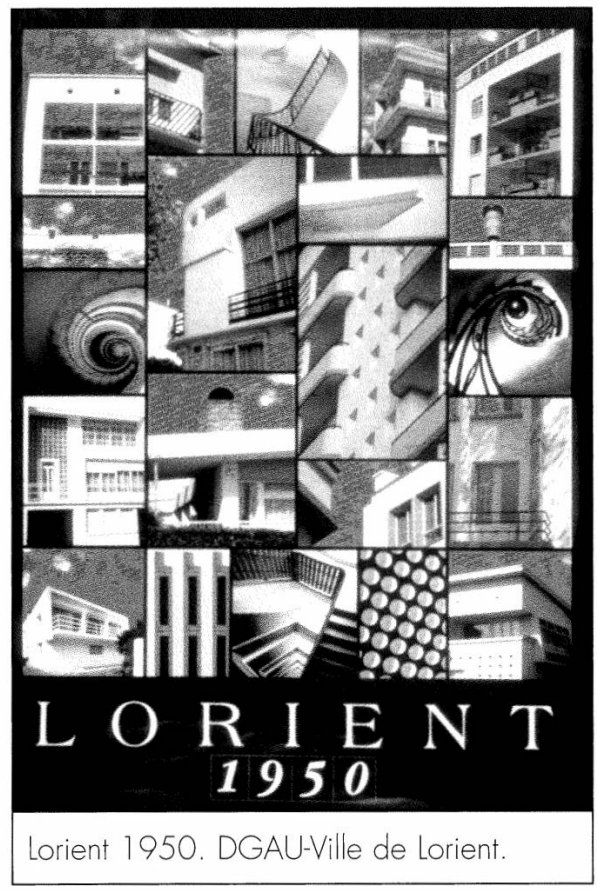

Du point de vue du tourisme urbain luimême, l'offre est encore embryonnaire. Le parcours « une ville des années cinquante» figure en bonne place dans les catalogues distribués à la presse, aux touristes et aux opérateurs, mais ce parcours n'est guère suivi que par une mince fraction des milliers de visiteurs qu'attire la base de sous-marins. Il faut néanmoins ajouter à cette statistique, qui pourrait apparaître d'autant plus décourageante qu'elle concerne essentiellement un public local, les nombreuses visites scolaires et universitaires, et un public de professionnels de l'urbanisme et, plus récemment, du tourisme ${ }^{6}$. Lorient a désormais vocation à devenir l'un des lieux fondateurs

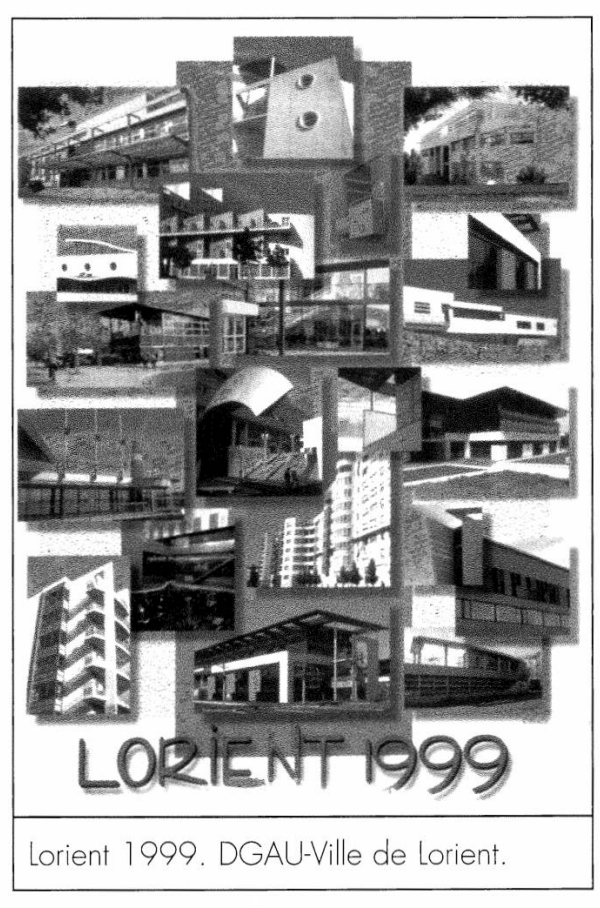

de la réflexion sur le patrimoine urbain contemporain, et sur son insertion dans l'offre touristique. Une convention entre le ministère de la Culture et la ville a permis de pérenniser cette orientation.

Enfin, il faut sans doute voir un signe de la conviction des élus lorientais, acquise de longue date, dans l'annonce qui vient d'être faite par le maire de Lorient, adjoint à l'urbanisme au moment des premières campagnes de réhabilitation : la mise en chantier d'un « centre d'interprétation de l'architecture du $\mathrm{XX}^{\mathrm{e}}$ siècle ». Ce pôle d'attraction fournira sans doute au tourisme urbain encore balbutiant le point d'ancrage

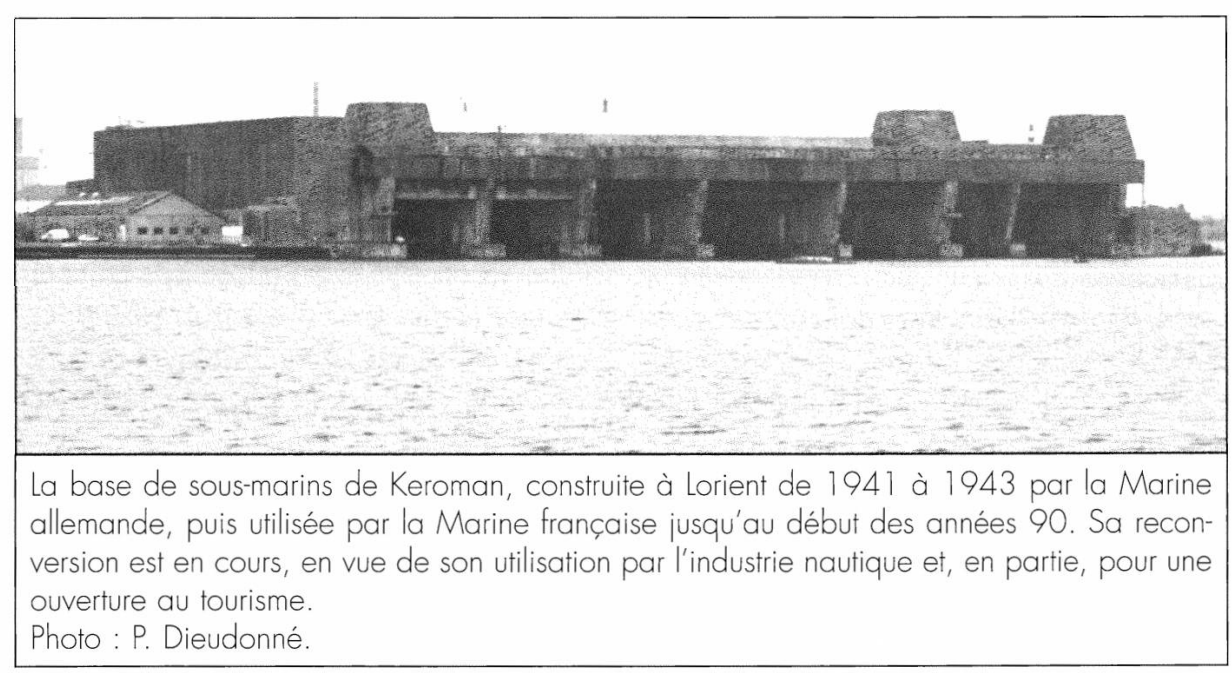




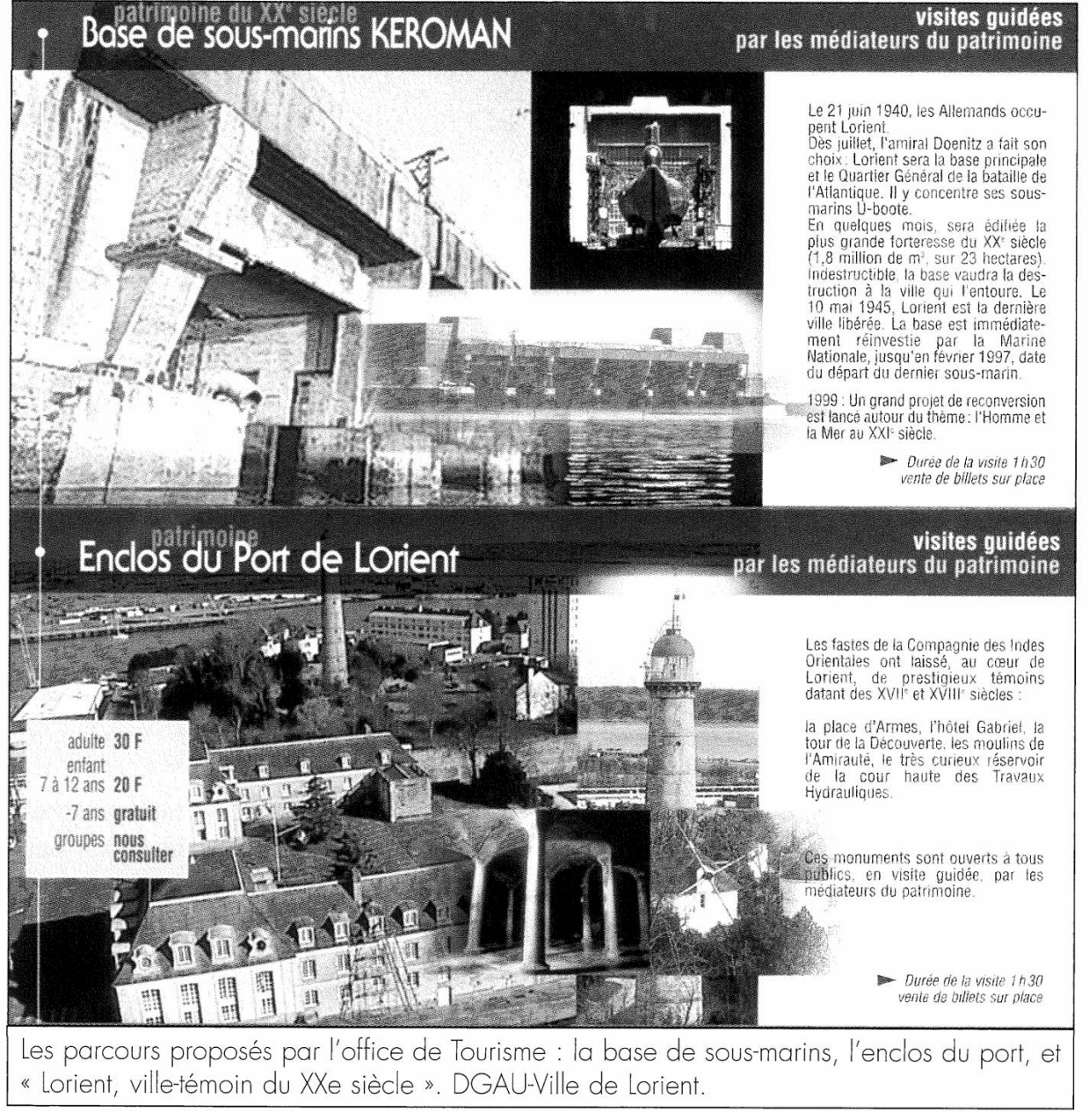

qui lui fait défaut, et dont la base de sousmarins, aussi spectaculaire soit-elle, ne remplit qu' imparfaitement le rôle.

L'intérêt de l'expérience lorientaise est sans doute d'avoir révélé deux caractéristiques du tourisme urbain. La nécessité d'associer étroitement la population à «l'invention » du patrimoine, tout d'abord. Loin d'avoir été une simple aventure intellectuelle, le colloque international des villes reconstruites, montrant aux habitants que des représentants de 22 pays s'intéressaient à une originalité que les Lorientais n'avaient guère soupçonnée, a permis une mobilisation dont certains ingrédients de l'offre touristique portent encore les fruits : ainsi, la visite de l'abri souterrain construit sous la place principale pendant la guerre, qui aurait pu être une simple célébration nostalgique, est aujourd'hui encore l'occasion de renforcer et de nuancer les fondements d'une mémoire urbaine. Le lien entre projet urbain et tourisme vient ensuite. Alors que la communication est volontiers schizophrène, séparant rigoureusement les campagnes de promotion touristiques et opérationnelles, la genèse de la réhabilitation des édifices a inscrit d'emblée le processus de construction de la notoriété au cœur de la diffusion elle-même. Aujourd'hui encore, des visiteurs venus de Caen ou du Havre, aux prises avec des problèmes semblables de valorisation patrimoniale, viennent chercher à Lorient conseils et méthodes. Ce tourisme professionnel gagnerait, du reste, à être renforcé, car à ce jour, rares sont les villes reconstruites qui ont su à ce point allier une campagne physique de réhabilitation à un travail aussi vigoureux et fructueux de sensibilisation au patrimoine contemporain.

Patrick Dieudonné est architecte et maître de conférences d'urbanisme à l'Institut de Géoarchitecture (Université de
Bretagne occidentale, Brest ). Ses travaux portent sur le patrimoine architectural et urbain du XX siècle, et notamment sur les créations urbaines ex nihilo, villes reconstruites et villes nouvelles. Les actes du colloque international des villes reconstruites sont rassemblés en deux volumes (Du dessin au destin, Paris :L'Harmattan, 1994).

\section{Notes}

1 Selon les statistiques officielles, 1838 communes ont été considérées comme sinistrées, représentant un total de $460000 \mathrm{im}$ meubles détruits et 1900000 immeubles endommagés. Cf. Danièle Voldman, La reconstruction des villes françaises de 1940 à 1954, Paris : L'Harmattan, 1997.

2 La loi française permet au maire d'une commune de contraindre, par arrêté municipal, les propriétaires à ravaler leur façade (nettoyage, réfection des enduits et peintures extérieures) tous les dix ans. Dans la pratique, cette contrainte laisse une large place à la négociation et un dispositif de subventions vient généralement la tempérer.

3 Introduit par les premières techniques de préfabrications à Orléans, le bloc-fenêtre se caractérise par un cadre en saillie sur la façade, dont l'épaisseur uniforme atteint couramment $10 \mathrm{~cm}$. À Lorient, il a fréquemment été peint de couleurs vives.

4 Zone de Protection du Patrimoine Architectural, Urbain et Paysager. Cet instrument juridique de protection, qui définit une zone d'intervention, un inventaire du patrimoine et des méthodes de protection des bâtiments, est l'équivalent des PIIA (Plans d'Implantation et d'Intégration Architecturale) dans la loi québécoise sur l'aménagement.

5 Selon les villes, la gestion des offices de tourisme peut être strictement municipale, parfois concédée en totalité ou en partie à des acteurs privés, ou organisée sur un territoire plus vaste correspondant à un palier d'administration regroupant plusieurs communes (communauté d'agglomération ou pays).

6 L'Institut régional du Patrimoine de Bretagne organise à Lorient des sessions de formation de guides-conférenciers sur le thème du tourisme urbain dans les quartiers contemporains. 